Document downloaded from:

http://hdl.handle.net/10251/65554

This paper must be cited as:

Artidiello Moreno, SDJ.; Cordero Barbero, A.; Torregrosa Sánchez, JR.; Vassileva, M. (2015). Two weighted-order classes of iterative root-finding methods. International Journal of Computer Mathematics. 92(9):1790-1805. doi:10.1080/00207160.2014.887201.

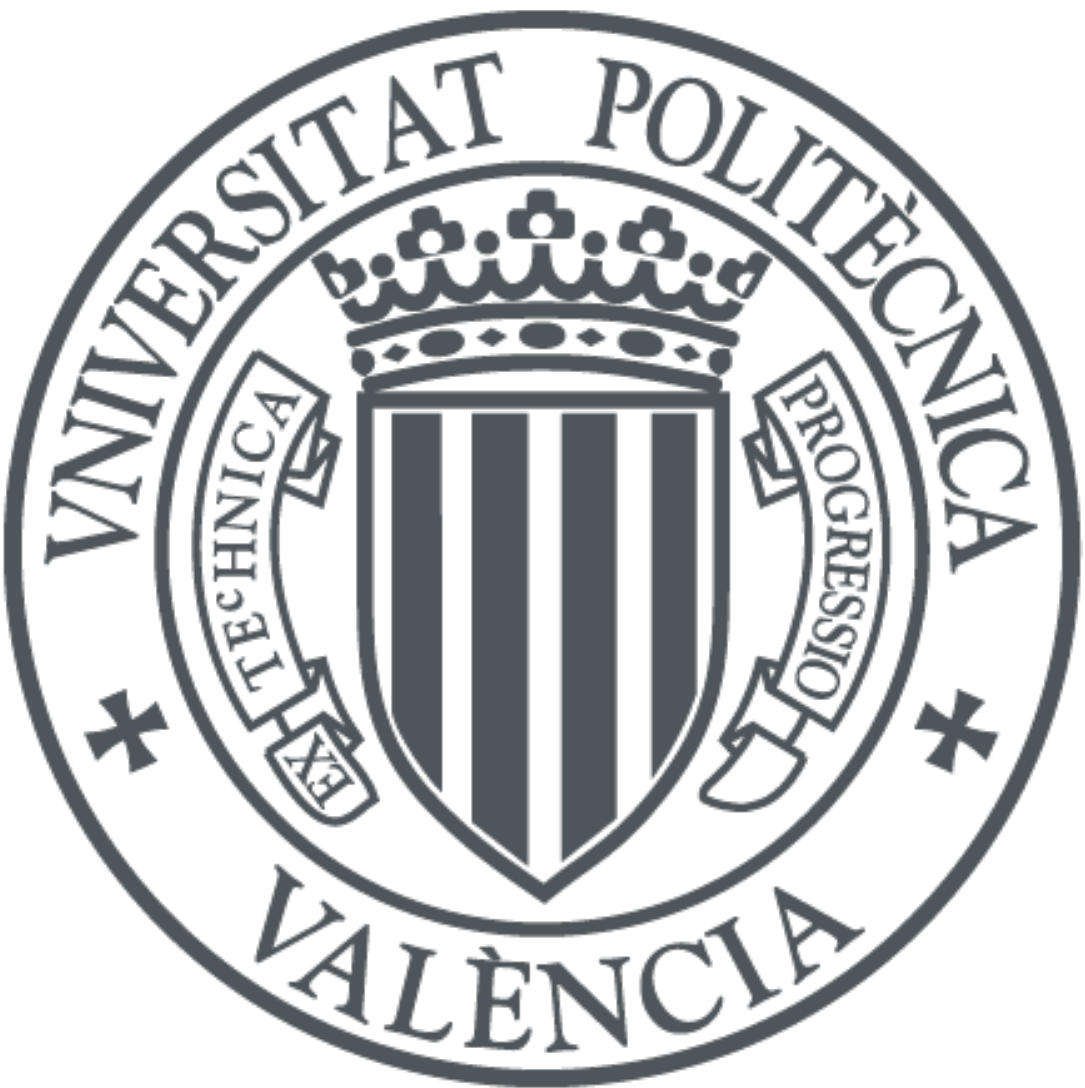

The final publication is available at

http://dx.doi.org/10.1080/00207160.2014.887201

Copyright Taylor \& Francis: STM, Behavioural Science and Public Health Titles

Additional Information 


\title{
Two weighted eight-order classes of iterative root-finding methods *
}

\author{
Santiago Artidiello ${ }^{1}$, Alicia Cordero ${ }^{2}$, Juan R. Torregrosa ${ }^{2}$ and María P. Vassileva ${ }^{1}$ \\ ${ }^{1}$ Instituto Tecnológico de Santo Domingo (INTEC), av. Los Próceres, Galá, Santo Domingo, República Dominicana. \\ ${ }^{2}$ Instituto de Matemática Multidisciplinar. Universitat Politécnica de Valencia. \\ santiago.artidiello@intec.edu.do, acordero@mat.upv.es, jrtorre@mat.upv.es, maria.penkova@intec.edu.do
}

\begin{abstract}
In this paper we design, by using the weight function technique, two families of iterative schemes with order of convergence eight. These weight functions depend on one, two and three variables and they are used in the second and third step of the iterative expression. Dynamics on polynomial and non-polynomial functions is analyzed and they are applied on the problem of preliminary orbit determination by using a modified Gauss method. Finally, some standard test functions are to check the reliability of the proposed schemes and allow us to compare them with other known methods.
\end{abstract}

Key Words: Nonlinear equation, iterative schemes, optimal methods, weight functions technique, efficiency index.

AMS 2000: 65H05, 65H10.

\section{Introduction}

Nonlinearity appears frequently in real problems, which are modeled by different branches of science and engineering. These models try to simplify the original problems holding their main properties. So, they usually remain nonlinear. The use of iterative techniques to estimate the solutions of these kind of problems has revealed itself as a fruitful area of research.

In general, we try to find a simple real solution $\xi \in I$ of a nonlinear equation $f(x)=0$. The most of the computationally useful techniques to determine roots can be classified as: (a) iterative methods that require only functional evaluations of $f$, and (b) methods whose iterative formula require evaluations of the function and its derivatives. There are two known simple and effective methods that represent these classes: Steffensen' and Newton's methods, respectively, both with order of convergence two. The search of variants of these methods with an accelerated convergence and a reduced number of operations and functional evaluations has resulted in the last decades in multistep methods. These schemes belong to the class of most powerful methods that overcome the limitations of the methods of a point with respect to the order of convergence and computational efficiency. This type of iterative methods were extensively studied in Traub's text, [1] and more recently, in [2].

The most of multistep iterative methods modify Newton's scheme to solve nonlinear equations with a highorder of convergence (see $[3,4,5,6,7]$ ). However, they frequently use derivatives, which is a serious disadvantage. Sometimes, the applications of iterative algorithms that depend on derivatives are restricted in engineering and science. For these cases, different authors have developed derivative-free iterative methods in numerous papers, that only need the additional evaluations of the function (see $[8,9,10,11])$.

In this paper (following the ideas presented in [12] and [13]) we design, in Section 2, two families of iterative methods belonging to classes (a) and (b), respectively, both of eighth-order of convergence. Their stability properties are tested on different functions in Section 3. In Section 4, an application is made on the problem of preliminary orbit determination. This problem is used to test the robustness and efficiency of the proposed methods, compared with other partners of the same order and with the original scheme used by Gauss and introduced in [14]. To get this aim, variable precision arithmetics is used to work with a high number of digits and a very restrictive stopping criterium which are not needed in real cases. Finally, in Section 5 some test

${ }^{*}$ This research was supported by Ministerio de Ciencia y Tecnología MTM2011-28636-C02-02 and FONDOCYT 2011-1-B1-33 República Dominicana 
functions are used to compare the numerical results of our methods with the obtained by other eighth-order schemes.

\section{Design of the families}

Let us remember that the iterative expression of Steffensen's scheme is

$$
x_{k+1}=x_{k}-\frac{f\left(x_{k}\right)}{f\left[z_{k}, x_{k}\right]},
$$

where $z_{k}=x_{k}+\beta f\left(x_{k}\right), f[.,$.$] denotes the usual first-order divided differences and Newton's one is$

$$
x_{k+1}=x_{k}-\frac{f\left(x_{k}\right)}{f^{\prime}\left(x_{k}\right)} .
$$

If we compose any of these methods with itself, schemes of order 4 with 4 functional evaluations are obtained in both cases. That makes them non-optimal, according to the conjecture of Kung-Traub [15].

In double Newton scheme, let us consider a frozen derivative in the second step, obtaining the non-optimal Traub's method of order 3 (see [1]). The authors in [13], using the technique of weight functions, got to increase to four the order of convergence of the Traub's scheme without adding new functional evaluations. The iterative expression of the obtained method is

$$
\begin{aligned}
y_{k} & =x_{k}-\frac{f\left(x_{k}\right)}{f^{\prime}\left(x_{k}\right)}, \\
x_{k+1} & =y_{k}-H\left(u_{k}\right) \frac{f\left(y_{k}\right)}{f^{\prime}\left(x_{k}\right)},
\end{aligned}
$$

where $H\left(u_{k}\right)$ represents a real-valued function and $u_{k}=\frac{f\left(y_{k}\right)}{b_{1} f\left(x_{k}\right)+b_{2} f\left(y_{k}\right)}$, being $b_{1}$ and $b_{2}$ real parameters.

Now, let us consider the double Steffensen scheme, holding the divided difference in the denominator in the second step. By using the technique of weight functions, Petković et al. in [12] increase to four the order of convergence of double Steffensen method without adding new functional evaluations. The iterative scheme of the resulting method is

$$
\begin{aligned}
y_{k} & =x_{k}-\frac{f\left(x_{k}\right)}{f\left[z_{k}, x_{k}\right]}, \\
x_{k+1} & =y_{k}-H\left(u_{k}, v_{k}\right) \frac{f\left(y_{k}\right)}{f\left[z_{k}, x_{k}\right]},
\end{aligned}
$$

where $z_{k}=x_{k}+\beta f\left(x_{k}\right), u_{k}=\frac{f\left(y_{k}\right)}{f\left(x_{k}\right)}$ and $v_{k}=\frac{f\left(y_{k}\right)}{f\left(z_{k}\right)}$. Under some conditions on $H$ and for nonzero arbitrary values of $\beta,(2)$ is an optimal method of order four.

Following these ideas, we design two classes of three-steps methods, that satisfy the following results. Let us notice that a particular subclass of the family presented in Theorem 1 was designed by Džunić et al. in [16].

Theorem 1 Let $\xi \in I$ be a simple zero of a sufficiently differentiable function $f: I \subset \mathbb{R} \rightarrow \mathbb{R}$ on an open interval I. Let $H$ and $G$ be sufficiently differentiable real functions and $x_{0}$ be an initial approximation close enough to $\xi$. If $\beta=1$ and $H$ and $G$ satisfy $H(0)=1, H^{\prime}(0)=2 b_{1}, H^{\prime \prime}(0)=2 b_{1}\left(2 b_{1}+b_{2}\right), G(0,0)=G(0,0)=1$, $G_{u}(0,0)=2 b_{1}, G_{u v}(0,0)=4 b_{1}, G_{u u}(0,0)=2 b_{1}\left(3 b_{1}+b_{2}\right)$ and $\left|G_{v v}(0,0)\right|<+\infty$. Then, the method

$$
\begin{aligned}
y_{k} & =x_{k}-\beta \frac{f\left(x_{k}\right)}{f^{\prime}\left(x_{k}\right)}, \\
z_{k} & =y_{k}-H\left(u_{k}\right) \frac{f\left(y_{k}\right)}{f^{\prime}\left(x_{k}\right)}, \\
x_{k+1} & =z_{k}-G\left(u_{k}, v_{k}\right) \frac{f\left(z_{k}\right)}{f^{\prime}\left(x_{k}\right)},
\end{aligned}
$$


where $u_{k}=\frac{f\left(y_{k}\right)}{b_{1} f\left(x_{k}\right)+b_{2} f\left(y_{k}\right)}$, being $b_{1}$ and $b_{2}$ arbitrary real parameters and $v_{k}=\frac{f\left(z_{k}\right)}{f\left(y_{k}\right)}$, has order of convergence eight for any value $b_{1}$ different from zero.

Proof. Let us introduce the Taylor's series expansion of $f$ and $f^{\prime}$ around $\xi$

$$
f\left(x_{k}\right)=f^{\prime}(\xi) \sum_{j=1}^{8} c_{j} e_{k}^{j}+O\left(e_{k}^{9}\right) \quad \text { and } \quad f^{\prime}\left(x_{k}\right)=f^{\prime}(\xi) \sum_{j=1}^{8}(j+1) c_{j+1} e_{k}^{j}+O\left(e_{k}^{9}\right)
$$

where $c_{j}=\frac{f^{(j)}(\xi)}{j ! f^{\prime}(\xi)}$ and $e_{k}=x_{k}-\xi$. Applying these developments on the damped Newton's method

$$
y_{k}=x_{k}-\beta \frac{f\left(x_{k}\right)}{f^{\prime}\left(x_{k}\right)}=\xi+\sum_{j=1}^{8} A_{j} e_{k}^{j}+O\left(e_{k}^{9}\right),
$$

where $A_{1}=1-\beta, A_{2}=-2 \beta c_{2}, A_{3}=2 \beta\left(c_{3}-c_{2}^{2}\right)$ and $A_{4}=\beta\left(4 c_{2}^{3}-7 c_{2} c_{3}+3 c_{4}\right)$. Using again Taylor's series expansion, we get

$$
f\left(y_{k}\right)=f^{\prime}(\xi) \sum_{j=1}^{8} B_{j} e_{k}^{j}+O\left(e_{k}^{9}\right)
$$

where $B_{1}=A_{1}, B_{2}=\left(\beta^{2}-\beta+1\right) c_{2}, B_{3}=-2 \beta^{2} c_{2}^{2}+\left(1-\beta+3 \beta^{2}-\beta^{3}\right) c_{3}$ and $B_{4}=\left(1-\beta+6 \beta^{2}-4 \beta^{3}+\right.$ $\left.\beta^{4}\right) c_{4}+5 \beta^{2} c_{2}^{3}+\beta^{2}(3 \beta-10) c_{3} c_{2}$.

Now, the expansion of $u_{k}=\frac{f\left(x_{k}\right)}{b_{1} f\left(x_{k}\right)+b_{2} f\left(y_{k}\right)}$ can be calculated and then, a Taylor's series expression is obtained for the weight function $H\left(u_{k}\right) \approx H(0)+H^{\prime}(0) u_{k}+(1 / 2) H^{\prime \prime}(0) u_{k}^{2}$. So,

$$
z_{k}=y_{k}-H\left(u_{k}\right) \frac{f\left(y_{k}\right)}{f^{\prime}\left(x_{k}\right)}=\xi+\sum_{j=1}^{8} Z_{j} e_{k}^{j}+O\left(e_{k}^{9}\right)
$$

and the error equation of the second step is $e_{z_{k}}=\sum_{j=1}^{8} Z_{j} e_{k}^{j}+O\left(e_{k}^{9}\right)$ whose first term depend on the factor

$$
Z_{1}=(1-\beta)\left[1-H(0)+\frac{1-\beta}{2\left(b_{1}+b_{2}(1-\beta)\right)^{2}}\left(-2 b_{1} H^{\prime}(0)+(\beta-1)\left(2 b_{2} H^{\prime}(0)+H^{\prime \prime}(0)\right)\right)\right] .
$$

The condition for obtaining at least convergence order 2 is $Z_{1}=0$, so we need $\beta=1$. Introducing this value in the error equation of this step we obtain $e_{z_{k}}=\sum_{j=2}^{8} \bar{Z}_{j} e_{k}^{j}+O\left(e_{k}^{9}\right)$, where

$$
\bar{Z}_{2}=(1-H(0)) c_{2}, \quad \text { and } \quad \bar{Z}_{3}=2(1-H(0)) c_{3}-\left(2-4 H(0)+H^{\prime}(0) / b_{1}\right) c_{2}^{2} .
$$

Solving the system of simultaneous equations $\bar{Z}_{2}=0$ and $\bar{Z}_{3}=0$ we get $H(0)=1$ and $H^{\prime}(0)=2 b_{1}$. Substituting these conditions we obtain the error expression

$$
e_{z_{k}}=\frac{1}{2 b_{1}^{2}}\left(\left(4 b_{1} b_{2}+10 b_{1}^{2}-H^{\prime \prime}(0)\right) c_{2}^{2}-2 b_{1}^{2} c_{3}\right) c_{2} e_{k}^{4}+\sum_{j=5}^{8} \overline{\bar{Z}}_{j} e_{k}^{j}+O\left(e_{k}^{9}\right) .
$$

From this expression we conclude that the second step of the iterative expression (3) can have convergence order no more than 4 as $b_{1} \neq 0$. By using again Taylor's expansion $f(z)=f^{\prime}(\xi) \sum_{j=4}^{8} D_{j} e_{k}^{j}+O\left(e_{k}^{9}\right)$, where $D_{4}=\frac{1}{2 b_{1}^{2}}\left(\left(4 b_{1} b_{2}+10 b_{1}^{2}-H^{\prime \prime}(0)\right) c_{2}^{2}-2 b_{1}^{2} c_{3}\right) c_{2}$. 
Now, we define the new variable $v_{k}=\frac{f\left(z_{k}\right)}{f\left(y_{k}\right)}$ and the weight function $G\left(u_{k}, v_{k}\right) \approx G(0,0)+G_{u}(0,0) u_{k}+$ $G_{v}(0,0) v_{k}+\frac{1}{2}\left(G_{u u}(0,0) u_{k}^{2}+G_{v v}(0,0) v_{k}^{2}\right)+G_{u v}(0,0) u_{k} v_{k}$.

Then, as $x_{k+1}=z_{k}-G\left(u_{k}, v_{k}\right) \frac{f\left(z_{k}\right)}{f^{\prime}\left(x_{k}\right)}$ we obtain

$$
e_{k+1}=\frac{1}{2 b_{1}^{2}}(1-G(0,0))\left[\left(4 b_{1} b_{2}+10 b_{1}^{2}-H^{\prime \prime}(0)\right) c_{2}^{2}-2 b_{1}^{2} c_{3}\right] c_{2} e_{k}^{4}+\sum_{j=5}^{8} L_{j} e_{k}^{j}+O\left(e_{k}^{9}\right) .
$$

If we choose $G(0,0)=1$, the order of convergence will be at least 5 . Substituting these condition in the error equation we obtain the new expression of it

$$
e_{k+1}=\frac{1}{2 b_{1}^{3}}\left(2 b_{1}-G_{u}(0,0)\right)\left[\left(4 b_{1} b_{2}+10 b_{1}^{2}-H^{\prime \prime}(0)\right) c_{2}^{2}-2 b_{1}^{2} c_{3}\right] c_{2} e_{k}^{5}+\sum_{j=6}^{8} \bar{L}_{j} e_{k}^{j}+O\left(e_{k}^{9}\right) .
$$

Let us observe that if $G_{u}(0,0)=2 b_{1}$, the term of order 5 vanishes. Again, we substitute this condition in the error equation and simplifying we obtain

$$
e_{k+1}=\sum_{j=6}^{8} \overline{\bar{L}}_{j} e_{k}^{j}+O\left(e_{k}^{9}\right)
$$

where $\overline{\bar{L}}_{6}=\frac{1}{4 b_{1}^{4}}\left(\left(4 b_{1} b_{2}+10 b_{1}^{2}-H^{\prime \prime}(0)\right) c_{2}^{2}-2 b_{1}^{2} c_{3}\right)\left[\left(r_{1} c_{2}^{2}+2 b_{1}^{2}\left(1-G_{v}(0,0)\right) c_{3}\right] c_{2}\right.$ and $r_{1}=4 b_{1} b_{2}\left(G_{v}(0,0)-\right.$ $1)+2 b_{1}^{2}\left(5 G_{v}(0,0)-6\right)+G_{u u}(0,0)-G_{v}(0,0) H^{\prime \prime}(0)$. If $G_{v}(0,0)=1$ and $G_{u u}(0,0)=2 b_{1}^{2}+H^{\prime \prime}(0)$ the order of convergence is at least 7 and the error expression obtained $e_{k+1}=\overline{\bar{L}}_{7} e_{k}^{7}+\overline{\bar{L}}_{8} e_{k}^{8}+O\left(e_{k}^{9}\right)$, where

$$
\overline{\bar{L}}_{7}=\frac{1}{4 b_{1}^{5}}\left(n c_{2}^{2}-2 b_{1}^{2} c_{3}\right)\left[r_{2} c_{2}^{2}-\left(8 b_{1}^{3}+2 b_{1}^{2} G_{u v}(0,0)\right) c_{3}\right) c_{2}^{2} .
$$

In order to reach eighth-order of convergence we need $\overline{\bar{L}}_{7}=0$, that is, $G_{u v}(0,0)=4 b_{1}$ and $H^{\prime \prime}(0)=2\left(2 b_{1}^{2}+b_{1} b_{2}\right)$.

Finally,

$$
e_{k+1}=\frac{1}{2 b_{1}^{3}}\left[\left(3 b_{1}+b_{2}\right) c_{2}^{2}-b_{1} c_{3}\right]\left[r_{3} c_{3}^{2}+r_{4} c_{2}^{4}+r_{5} c_{2}^{2} c_{3}-2 b_{1}^{2} c_{2} c_{4}\right] e_{k}^{8}+O\left(e_{k}^{9}\right),
$$

where $r_{3}=b_{1}^{2}\left(G_{v v}-2\right), r_{4}=9 b_{1}^{2}\left(G_{v v}-6\right)+b_{2}^{2}\left(G_{v v}-2\right)+2 b_{1} b_{2}\left(3 G_{v v}-13\right)$ and $r_{5}=b_{1}^{2}\left(34-G_{v v}\right)-2 b_{1} b_{2}\left(G_{v v}-5\right)$ and the proof of the result is finished.

By choosing different weight functions verifying the conditions imposed in Theorem1, different classes of methods can be found. Specifically, in the next sections we will use

$$
\begin{aligned}
H(u) & =1+2 b_{1} u+b_{1}\left(2 b_{1}+b_{2}\right) u^{2}, \\
G(u, v) & =1+2 b_{1} u+v+b_{1}\left(3 b_{1}+b_{2}\right) u^{2}+4 b_{1} u v .
\end{aligned}
$$

Let us note that if $b_{1}=1$ and $b_{2}=-2$ then $H(u)=1+2 u$ and the first two steps of (3) correspond to the Ostrowski's method. In this case function $G$ is $G(u, v)=1+2 u+v+u^{2}+4 u v$.

Respect to the class of derivative-free methods, mentioned in the Introduction, and taking into account Petković 's idea showed in (2), we establish the following result.

Theorem 2 Let $\xi \in I$ be a simple zero of a sufficiently differentiable function $f: I \subset \mathbb{R} \rightarrow \mathbb{R}$ on an open interval $I$ and be $x_{0}$ an initial approximation close enough to $\xi$. Let $H$ and $G$ be sufficiently differentiable real functions which satisfy: $H(0,0)=H_{u}(0,0)=H_{v}(0,0)=1, H_{u u}(0,0)=H_{v v}(0,0)=2, H_{u v}(0,0)=0, G(0,0,0)=$ $G_{u}(0,0,0)=G_{v}(0,0,0)=G_{w}(0,0,0)=1, G_{u u}(0,0,0)=G_{v v}(0,0,0)=G_{u w}(0,0,0)=G_{v w}(0,0,0)=2$, $G_{u v}(0,0,0)=1$ and $\left|G_{w w}(0,0,0)\right|<\infty$. Then, the method

$$
\begin{aligned}
y_{k} & =x_{k}-\frac{f\left(x_{k}\right)}{f\left[z_{k}, x_{k}\right]}, \\
t_{k} & =y_{k}-H\left(u_{k}, v_{k}\right) \frac{f\left(y_{k}\right)}{f\left[z_{k}, x_{k}\right]}, \\
x_{k+1} & =t_{k}-G\left(u_{k}, v_{k}, w_{k}\right) \frac{f\left(t_{k}\right)}{f\left[z_{k}, x_{k}\right]},
\end{aligned}
$$


where $z_{k}=x_{k}+\beta f\left(x_{k}\right), u_{k}=\frac{f\left(y_{k}\right)}{f\left(x_{k}\right)}, v_{k}=\frac{f\left(y_{k}\right)}{f\left(z_{k}\right)}$ and $w_{k}=\frac{f\left(t_{k}\right)}{f\left(y_{k}\right)}$, has order of convergence eight for any value $\beta$ different from zero and the error equation is

$$
\begin{aligned}
e_{k+1} & =-\frac{1}{2} \rho^{2} c_{2}\left(3 c_{2}^{2}-c_{3}\right)\left(\left(9 G_{w w}(0,0,0) \rho^{2}+2\left(-27-54 \gamma-28 \gamma^{2}+\gamma^{3}\right)+\gamma^{4}\right)\right) c_{2}^{4}+2 \rho\left(17+17 \gamma+2 \gamma^{2}\right. \\
& \left.\left.\left.-3 G_{w w}(0,0,0) \rho\right) c_{2}^{2} c_{3}+\left(-2+G_{w w}(0,0,0)\right) \rho^{2} c_{3}^{2}-2 \rho^{2} c_{2} c_{4}\right)\right) e_{k}^{8}+O\left(e_{k}^{9}\right),
\end{aligned}
$$

where $\rho=1+\gamma$ and $\gamma=f^{\prime}(\xi) \beta$.

Proof. By using the Taylor's series expansion of $f\left(x_{k}\right)$ introduced in the previous theorem, we have

$$
z_{k}=\rho e_{k}+\gamma \sum_{j=2}^{8} c_{j} e_{k}^{j}+O\left(e_{k}^{9}\right) .
$$

So,

$$
f\left(z_{k}\right)=f^{\prime}(\xi) \sum_{j=1}^{8} Z_{j} e_{k}^{j}+O\left(e_{k}^{9}\right)
$$

where $Z_{1}=\rho, Z_{2}=\left(\gamma+\rho^{2}\right) c_{2}, Z_{3}=2 \gamma \rho c_{2}^{2}+\left(\gamma+\rho^{3}\right) c_{3}$ and $Z_{4}=3 \gamma \rho^{2} c_{2} c_{3}+\gamma c_{2}\left(\gamma c_{2}^{2}+2 \rho c_{3}\right)+\left(\gamma+\rho^{4}\right) c_{4}$; and,

$$
f\left[x_{k}, z_{k}\right]=\frac{f\left(z_{k}\right)-f\left(x_{k}\right)}{z_{k}-x_{k}}=f^{\prime}(\xi)\left[1+\sum_{j=1}^{8} D_{j} e_{k}^{j}+\right]+O\left(e_{k}^{9}\right),
$$

where $D_{1}=(1+\rho) c_{2}, D_{2}=\gamma c_{2}^{2}+\left(3 \rho+\gamma^{2}\right) c_{3}$ and $D_{3}=f^{\prime}(\xi)(1+\rho)\left(2 \gamma c_{2} c_{3}+\left(2 \rho+\gamma^{2}\right) c_{4}\right)$.

Therefore, the error at the first step of the method is

$$
e_{y_{k}}=y_{k}-\xi=\sum_{j=2}^{8} Y_{j} e_{k}^{j}+O\left(e_{k}^{9}\right),
$$

where $Y_{2}=\rho c_{2}, Y_{3}=-\left(2 \rho+\gamma^{2}\right) c_{2}^{2}+\left(2+3 \gamma+\gamma^{2}\right) c_{3}$ and $Y_{4}=\left(4+5 \gamma+3 \gamma^{2}+\gamma^{3}\right) c_{2}^{3}-\left(7+10 \gamma+7 \gamma^{2}+2 \gamma^{3}\right) c_{2} c_{3}+$ $\left(3+6 \gamma+4 \gamma^{2}+\gamma^{3}\right) c_{4}$.

Let us calculate the Taylor's expressions for the variables $u_{k}=\frac{f\left(y_{k}\right)}{f\left(x_{k}\right)}$ and $v_{k}=\frac{f\left(y_{k}\right)}{f\left(z_{k}\right)}$ and the weight function $H$ around $(0,0)$

$$
H\left(u_{k}, v_{k}\right) \approx H(0,0)+H_{u}(0,0) u_{k}+H_{v}(0,0) v_{k}+\frac{1}{2} H_{u u}(0,0) u_{k}^{2}+\frac{1}{2} H_{v v}(0,0) v_{k}^{2}+H_{u v}(0,0) u_{k} v_{k} .
$$

Then, the error equation of the second step is

$$
e_{t_{k}}=t_{k}-\xi=\sum_{j=2}^{8} R_{j} e_{k}^{j}+O\left(e_{k}^{9}\right)
$$

where $R_{2}=-(-1+H(0,0)) \rho c_{2}$ and $R_{3}=-\left(2 \rho+H_{v}(0,0)+\gamma H_{v}(0,0)+\gamma^{2}+\rho^{2} H_{u}(0,0)-H(0,0)(4+5 \gamma+\right.$ $\left.\left.2 \gamma^{2}\right)\right) c_{2}^{2}-(-1+H(0,0))\left(2+3 \gamma+\gamma^{2}\right) c_{3}$. In order to $t_{k}$ reaches fourth-order of convergence, we need to assume that $H(0,0)=H_{u}(0,0)=H_{v}(0,0)=1$. With these values,

$$
e_{t_{k}}=\sum_{j=4}^{8} \bar{R}_{j} e_{k}^{j}+O\left(e_{k}^{9}\right)
$$

where $\bar{R}_{4}=-\frac{1}{2} \rho\left(r_{4,1} c_{2}^{3}+r_{4,2} c_{2} c_{3}\right), r_{4,1}=-2\left(5 \rho+\gamma^{2}\right)+h_{v v}(0,0)+2 h_{u v}(0,0) \rho+h_{u u}(0,0) \rho^{2}$ and $r_{4,2}=2 \rho$. Then,

$$
f\left(t_{k}\right)=f^{\prime}(\xi) \sum_{j=4}^{8} \bar{R}_{j} e_{k}^{j}+O\left(e_{k}^{9}\right)
$$


Now, we calculate the expression of $w_{k}=\frac{f\left(t_{k}\right)}{f\left(y_{k}\right)}$ and the expression of the weight function $G\left(u_{k}, v_{k}, w_{k}\right)$ around $(0,0,0)$

$$
\begin{aligned}
G\left(u_{k}, v_{k}, w_{k}\right) & \approx G(0,0,0)+G_{u}(0,0,0) u_{k}+G_{v}(0,0,0) v_{k}+G_{w}(0,0,0) w_{k}+\frac{1}{2} G_{u u}(0,0,0) u_{k}^{2}+\frac{1}{2} G_{v v}(0,0,0) v_{k}^{2} \\
& +\frac{1}{2} G_{w w}(0,0,0) w_{k}^{2}+G_{u v}(0,0,0) u_{k} v_{k}+G_{u w}(0,0,0) u_{k} w_{k}+G_{v w}(0,0,0) v_{k} w_{k}
\end{aligned}
$$

Therefore, the error equation of the method is

$$
e_{k+1}=\sum_{j=4}^{8} L_{j} e_{k}^{j}+O\left(e_{k}^{9}\right),
$$

where $L_{4}=\frac{1}{2}(-1+G(0,0,0)) \rho\left(r_{4,1} c_{2}^{3}+r_{4,2} c_{2} c_{3}\right)$. If we assume $G(0,0,0)=1$, then the fourth-order term vanishes and $\bar{L}_{5}=\frac{1}{2}\left(-2+G_{u}(0,0,0)+G_{v}(0,0,0)-\gamma\left(1-G_{u}(0,0,0)\right)\right) \rho\left(r_{4,1} c_{2}^{4}+r_{4,2} c_{2}^{2} c_{3}\right)$.

Now, if $G_{u}(0,0,0)=G_{v}(0,0,0)=1$, we reach the sixth-order and $\overline{\bar{L}}_{6}=\frac{1}{4} \rho\left(r_{4,1} c_{2}^{3}+r_{4,2} c_{2} c_{3}\right)\left(\left(-2\left(6 \rho+\gamma^{2}\right)+\right.\right.$ $2 G_{u v}(0,0,0) \rho+G_{v v}(0,0,0)+G_{u u}(0,0,0) \rho^{2}+G_{w}(0,0,0)\left(2\left(5 \rho^{2}+\gamma^{2}\right)-H_{u u}(0,0) \rho^{2}-2 H_{u v}(0,0) \rho-H_{v v}(0,0)\right) c_{2}^{3}-$ $\left.2\left(G_{w}(0,0,0)-1\right) \rho c_{2} c_{3}\right)$.

Again, by imposing conditions $G_{u v}(0,0,0)=1+H_{u v}(0,0), G_{w}(0,0,0)=1$ and $H_{u u}(0,0)=G_{u u}(0,0,0)$, we obtain order 7 with

$$
\begin{aligned}
\overline{\bar{L}}_{7}= & -\frac{1}{4}\left(\rho c_{2}^{2}\left(\left(-10+2 H_{u v}(0,0)+H_{v v}(0,0)-10 \gamma+2 \gamma H_{u v}(0,0)-2 \gamma^{2}+G_{u u}(0,0,0) \rho^{2}\right) c_{2}^{2}+2 \rho c_{3}\right)\right. \\
& \left(\left(32-10 G_{v w}(0,0,0)-4 H_{u v}(0,0)+2 G_{v w}(0,0,0) H_{u v}(0,0)-2 H_{v v}(0,0)+G_{v w}(0,0,0) H_{v v}(0,0)\right.\right. \\
& +48 \gamma-10 \gamma G_{v w}(0,0,0)-6 \gamma H_{u v}(0,0)+2 \gamma G_{v w}(0,0,0) H_{u v}(0,0)-\gamma H_{v v}(0,0)+20 \gamma^{2} \\
& -2 \gamma^{2} G_{v w}(0,0,0)-2 \gamma^{2} H_{u v}(0,0)+2 \gamma^{3}+G_{u u}(0,0,0) \rho^{2}\left(-2+G_{u w}(0,0,0)+G_{v w}(0,0,0)\right. \\
& \left.\left.-\gamma+\gamma G_{u w}(0,0,0)\right)+G_{u w}(0,0,0) \rho\left(H_{v v}(0,0)+2 H_{u v}(0,0) \rho-2\left(5+5 \gamma+\gamma^{2}\right)\right)\right) c_{2}^{2} \\
& \left.\left.+2 \rho\left(-4+G_{u w}(0,0,0)+G_{v w}(0,0,0)-2 \gamma+\gamma G_{u w}(0,0,0)\right) c_{3}\right)\right)
\end{aligned}
$$

Finally, conditions $G_{u u}(0,0,0)=G_{u w}(0,0,0)=G_{v w}(0,0,0)=2, H_{u v}(0,0)=0$ and $H_{v v}(0,0)=2$, allows us to gain eighth-order with the following error equation

$$
\begin{aligned}
e_{k+1} & =-\frac{1}{2} \rho^{2} c_{2}\left(3 c_{2}^{2}-c_{3}\right)\left(\left(9 G_{w w}(0,0,0) \rho^{2}+2\left(-27-54 \gamma-28 \gamma^{2}+\gamma^{3}\right)+\gamma^{4}\right)\right) c_{2}^{4}+2 \rho\left(17+17 \gamma+2 \gamma^{2}\right. \\
& \left.\left.\left.-3 G_{w w}(0,0,0) \rho\right) c_{2}^{2} c_{3}+\left(-2+G_{w w}(0,0,0)\right) \rho^{2} c_{3}^{2}-2 \rho^{2} c_{2} c_{4}\right)\right) e_{k}^{8}+O\left(e_{k}^{9}\right)
\end{aligned}
$$

and the proof is finished.

Remark 1 The families of three-point methods (3) and (5) require four functional evaluations and have order of convergence eight. Therefore, these families are optimal in the sense of the Kung-Traub conjecture and have computational efficiency index $8^{1 / 4} \approx 1.6818$.

In this case, some of the functions $H$ and $G$ satisfying conditions of Theorem 2 are

$$
\begin{aligned}
H(u, v) & =1+u+v+u^{2}+v^{2} \\
G(u, v, w) & =1+u+v+w+u^{2}+v^{2}+u v+2(v w+u w) .
\end{aligned}
$$

In the following sections we are going to use the elements of the families (3), for $b_{1}=1$ and $b_{2}=0$, and (5) obtained by choosing the weight functions (4) and (12). These elements of the class will be denoted by $M_{1} 8$ and $M_{2} 8$, respectively.

\section{Dynamical aspects}

The dynamical properties of the rational function associated to an iterative method acting on a polynomial give us important information about numerical features of the method as its stability and reliability (see [17]). 
The dynamical behavior of the orbit of a point on the complex plane can be classified depending on its asymptotic behavior. In this way, a point in the Riemann sphere $z_{0} \in \hat{\mathbb{C}}$ is a fixed point of $R$ if $R\left(z_{0}\right)=z_{0}$. A fixed point is attracting, repelling or parabolic if $\left|R^{\prime}\left(z_{0}\right)\right|$ is less than, greater than or equal to 1 , respectively. Moreover, if $\left|R^{\prime}\left(z_{0}\right)\right|=0$, the fixed point is superattracting.

If $z^{*}$ is an attracting fixed point of the rational function $R$, its basin of attraction $\mathcal{A}\left(z^{*}\right)$ is defined as the set of pre-images of any order such that

$$
\mathcal{A}\left(z_{f}^{*}\right)=\left\{z_{0} \in \hat{\mathbb{C}}: R^{n}\left(z_{0}\right) \rightarrow z^{*}, n \rightarrow \infty\right\}
$$

The set of points whose orbits tends to an attracting fixed point $z_{f}^{*}$ is defined as the Fatou set, $\mathcal{F}(R)$. The complementary set in the Riemann sphere, the Julia set $\mathcal{J}(R)$, is the closure of the set consisting of its repelling fixed points, and establishes the borders between the basins of attraction.

For the representation of the convergence basins of every iterative procedure we have used the software described in [18]. In order to draw the dynamical planes, each point of the complex plane is considered as a starting point of the iterative scheme and it is painted in different colors depending on the point which it has converged to (the roots of the polynomial are marked in the figures by white stars). The figures has been generated for values of $z_{0}$ in $[-2,2] \times[-2,2]$, with a mesh of $800 \times 800$ points and 80 iterations per point. Depending on the number of iterations needed to converge, the color of the starting point will be brighter (less iterations) or darker (more iterations). We will represent the dynamical behavior of the mentioned elements of the two suggested classes, (3) and (5), for low-degree polynomials and non-polynomial functions, showing their stability and the amplitude of the convergence regions in these cases.

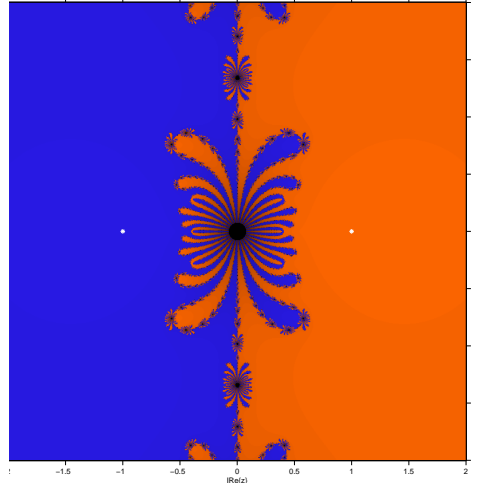

(a) $x^{2}-1$

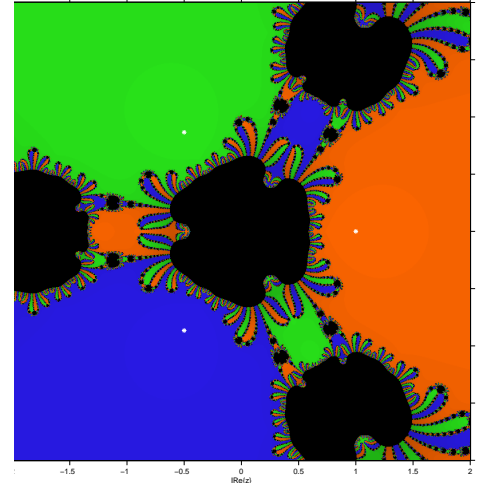

(b) $x^{3}-1$

Figure 1: Dynamical planes for the scheme $M_{1} 8$

When $M_{1} 8$ is considered, we observe in Figure 1 the basin of attraction of the different roots of low-degree polynomials. It is possible to observe some regions of slow convergence, in form of "flowers", whose petals make narrower while the convergence is slower. The black point in the center of the flowers correspond to regions of no convergence. The observed dynamical planes define wide amplitudes for the different basins of the roots.

If we consider now the rational function associated to the element of family (5) and analyze its dynamical behavior, we find that there exist black regions in the dynamical planes (see Figure 2) whose orbits do not tend to any of the roots, but to the infinity. So, the are wide regions of no convergence. Moreover, it can be observed that the amplitude of the convergence regions is narrower in some cases. Nevertheless, the convergence improves when $\beta$ is closer to zero than when it is near one.

In Figure 3, we show the dynamical planes corresponding to function $f(x)=10 x e^{-x^{2}}-1$ in $[-2,2] \times[-2,2]$, where two simple roots appear. These planes have been obtained by using Newton, $M_{1} 8$ and $M_{2} 8$ methods. In all cases, it can be observed that wide regions of non convergence appear. Some of them are due to poles of the function $f$. We observe that the behavior of the proposed methods is not very different from the one of Newton's scheme. 


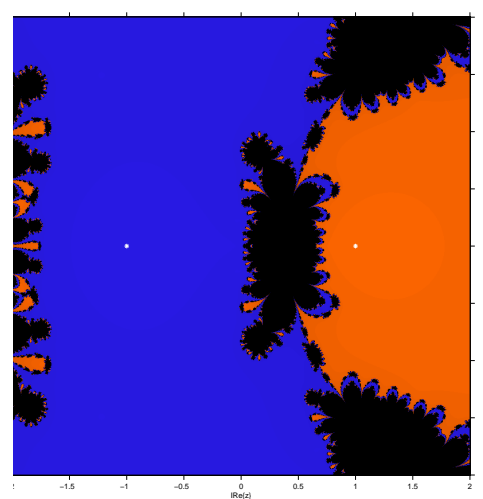

(a) $x^{2}-1, \beta=0.9$

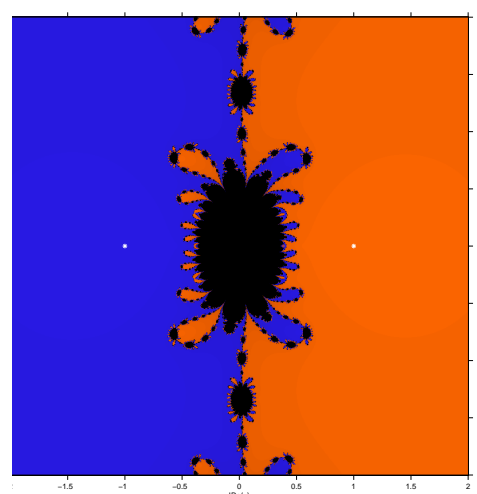

(b) $x^{2}-1, \beta=0.01$

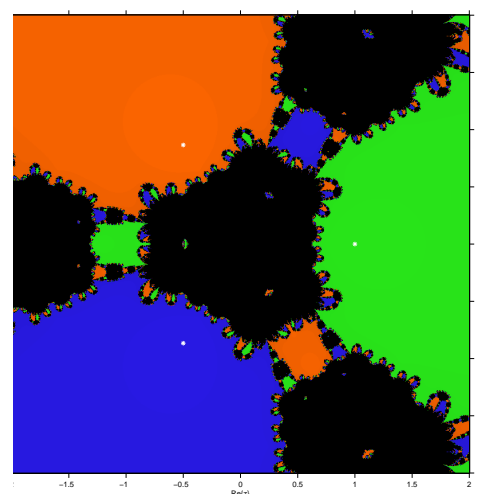

(c) $x^{3}-1, \beta=0.01$

Figure 2: Dynamical planes for the method $M_{2} 8$

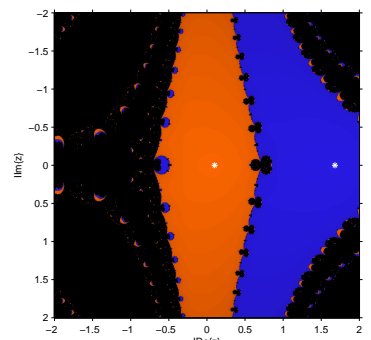

(a) Newton

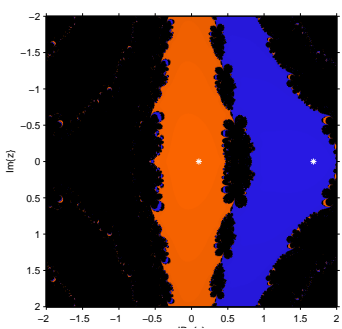

(b) $M_{1} 8$

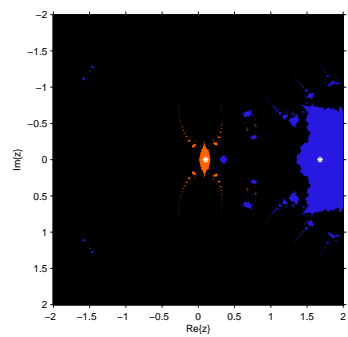

(c) $M_{2} 8, \beta=0.9$

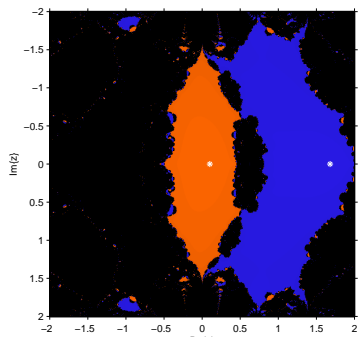

(d) $M_{2} 8, \beta=0.01$

Figure 3: Dynamical planes for function $10 x e^{-x^{2}}-1$

\section{Preliminary orbit determination}

A classical reference in preliminary orbit determination is F. Gauss (1777-1855), who deduced the orbit of the minor planet Ceres, discovered in 1801 and afterwards lost. The calculation of its trajectory by means of the procedure designed by Gauss and the re-discovering in following Gauss' calculations, marked the international recognition of Gauss and his work.

The so-called Gauss' method is based on the rate $y$ between the triangle and the ellipse sector defined by two position vectors, from astronomical observations. This proportion is related with the geometry of the orbit and the observed position by

$$
y=1+X(l+x)
$$

where

and

$$
l=\frac{r_{1}+r_{2}}{4 \sqrt{r_{1} r_{2}} \cos \left(\frac{\nu_{2}-\nu_{1}}{2}\right)}-\frac{1}{2}, \quad x=\sin ^{2}\left(\frac{E_{2}-E_{1}}{4}\right),
$$

$$
X=\frac{E_{2}-E_{1}-\sin \left(E_{2}-E_{1}\right)}{\sin ^{3}\left(\frac{E_{2}-E_{1}}{2}\right)} .
$$

The angles $E_{i}, \nu_{i}, i=1,2$, are the eccentric and true anomalies, respectively, associated to the observed positions $\overrightarrow{r_{1}}$ and $\overrightarrow{r_{2}}$ (let us denote by $r_{i}$ the modulus of vector $\overrightarrow{r_{i}}, i=1,2$ ).

Equation (13) is, actually, the composition of the First Gauss Equation

$$
y^{2}=\frac{m}{l+x}
$$

and the Second Gauss Equation

$$
y^{2}(y-1)=m X
$$


where $m=\frac{\mu \tau^{2}}{\left[2 \sqrt{r_{1} r_{2}} \cos \left(\frac{\nu_{2}-\nu_{1}}{2}\right)\right]^{3}}, \mu$ is the gravitational parameter of the motion and $\tau$ is a modified time variable.

The original iterative procedure used to solve the nonlinear Gauss equation (13) is the Fixed Point method (see, for example, [14]) and is described in the following scheme:

(i) From the initial estimation $y_{0}=1, x_{0}=\frac{m}{y_{0}^{2}}-l$ is obtained (it is possible to calculate $m$ and $l$ from the observed positions $\overrightarrow{r_{1}}$ and $\overrightarrow{r_{2}}$ and the time $\tau$.

(ii) From $x_{0}$ and

$$
\cos \left(\frac{E_{2}-E_{1}}{2}\right)=1-2 x_{0}, \quad \sin \left(\frac{E_{2}-E_{1}}{2}\right)=+\sqrt{4 x_{0}\left(1-x_{0}\right)},
$$

we calculate $E_{2}-E_{1}$. Then, we obtain $X_{0}=\frac{E_{2}-E_{1}-\sin \left(E_{2}-E_{1}\right)}{\sin ^{3}\left(\frac{E_{2}-E_{1}}{2}\right)}$.

(iii) By using the combined Gauss equation (13)

$$
y_{1}=1+X_{0}\left(l+x_{0}\right),
$$

a new iteration is calculated and the process start again.

The iterative process follows as described above, getting new estimations of the ratio, until it does not vary within a given tolerance. Once the method has converged, the semi-major axis $a$, can be calculated by means of equation

$$
y=\frac{\sqrt{\mu p} \cdot \tau}{r_{2} r_{1} \sin \left(\nu_{2}-\nu_{1}\right)}=\frac{\sqrt{\mu} \cdot \tau}{2 \sqrt{a} \sqrt{r_{2} r_{1}} \sin \left(\frac{E_{2}-E_{1}}{2}\right) \cos \left(\frac{\nu_{2}-\nu_{1}}{2}\right)},
$$

from the last estimations of ratio and difference of eccentric anomalies, and the last phase is then initiated, to determine velocity and orbital elements.

Let us note that the initial estimation used is good only if the observations are close in time, so it is a weakness implicit in Gauss method. Indeed, the original fixed point scheme, with linear convergence, has been improved by the recent optimal high-order schemes. In particular, we are going to apply the proposed optimal eight-order methods, joint with some existing schemes of the same rate of convergence, in order to get an efficient modified-Gauss procedure of preliminary orbit determination, by solving the combined Gauss equation (13), with $y=1$ as an initial estimation.

All the iterative schemes introduced in the following are optimal in the sense of Kung-Traub's conjecture and have been designed with the weight-function technique, so they are fully comparable with the new ones designed in this paper. Let us refer now to the procedure that Kim present in [4]: a three-step eighth-order method, whose iterative expression is

$$
\begin{aligned}
y_{k} & =x_{k}-\frac{f\left(x_{k}\right)}{f^{\prime}\left(x_{k}\right)}, \\
z_{k} & =y_{k}-\frac{1+u_{k}+2 / 3 u_{k}^{2}}{1-u_{k}-2 u_{k}^{2}} \frac{f\left(y_{k}\right)}{f^{\prime}\left(x_{k}\right)}, \\
x_{k+1} & =z_{k}-\frac{1-2 u_{k}+v_{k}}{1-3 u_{k}-2 v_{k}} \frac{f\left(z_{k}\right)}{f^{\prime}\left(x_{k}\right)+f\left[y_{k}, x_{k}, z_{k}\right]\left(z_{k}-x_{k}\right)},
\end{aligned}
$$

where $u_{k}=\frac{f\left(y_{k}\right)}{f\left(x_{k}\right)}, v_{k}=\frac{f\left(z_{k}\right)}{f\left(x_{k}\right)}$ and $f[\cdot, \cdot, \cdot]$ denotes the divided difference of order two. We will denote this scheme by $\mathrm{K} 8$.

Another recent eighth-order method has been developed by Khan et. al. in [5]. Its iterative expression is:

$$
\begin{aligned}
y_{k} & =x_{k}-\frac{f\left(x_{k}\right)}{f^{\prime}\left(x_{k}\right)}, \\
z_{k} & =y_{k}-\frac{f\left(x_{k}\right)^{2}}{f\left(x_{k}\right)^{2}-2 f\left(x_{k}\right) f\left(y_{k}\right)+f\left(y_{k}\right)^{2}} \frac{f\left(y_{k}\right)}{f^{\prime}\left(x_{k}\right)}, \\
x_{k+1} & =z_{k}-\frac{1}{1+v_{k}^{2}} \frac{f\left(z_{k}\right)}{K-C\left(y_{k}-z_{k}\right)-D\left(y_{k}-z_{k}\right)^{2}},
\end{aligned}
$$


where $v_{k}=\frac{f\left(z_{k}\right)}{f\left(x_{k}\right)}, K=f\left[y_{k}, z_{k}\right], H=f\left[x_{k}, y_{k}\right], C=\frac{H-K}{\left(x_{k}-y_{k}\right)\left(x_{k}-z_{k}\right)}-D\left(x_{k}+y_{k}-2 z_{k}\right), D=\frac{f^{\prime}\left(x_{k}\right)-H}{\left(x_{k}-y_{k}\right)\left(x_{k}-z_{k}\right)}-$ $\frac{H-K}{\left(x_{k}-z_{k}\right)^{2}}$ and $f[\cdot, \cdot]$ denotes the divided difference of order 1 . In the following, we will denote this method by F8.

The third scheme that we will use to compare with, denoted by D8, was obtained by Džunić and Petković in [19]. It is based on Ostrowski's fourth-order scheme (see [20]) and its iterative expression is

$$
\begin{aligned}
y_{k} & =x_{k}-\frac{f\left(x_{k}\right)}{f^{\prime}\left(x_{k}\right)}, \\
z_{k} & =y_{k}-\frac{f\left(x_{k}\right)}{f\left(x_{k}\right)-2 f\left(y_{k}\right)} \frac{f\left(y_{k}\right)}{f^{\prime}\left(x_{k}\right)}, \\
x_{k+1} & =z_{k}-\frac{\left(1+w_{k}\right)\left(1+2 v_{k}\right)}{1-2 u_{k}-u_{k}^{2}} \frac{f\left(z_{k}\right)}{f^{\prime}\left(x_{k}\right)},
\end{aligned}
$$

where $u_{k}=\frac{f\left(y_{k}\right)}{f\left(x_{k}\right)}, v_{k}=\frac{f\left(z_{k}\right)}{f\left(x_{k}\right)}$ and $w_{k}=\frac{f\left(z_{k}\right)}{f\left(y_{k}\right)}$.

Finally, we will also compare our new schemes with the method designed by Soleymani et al. in [21] (denoted by S8), also initialized with Ostrowski's procedure,

$$
\begin{aligned}
y_{k} & =x_{k}-\frac{f\left(x_{k}\right)}{f^{\prime}\left(x_{k}\right)} \\
z_{k} & =y_{k}-\frac{f\left(x_{k}\right)}{f\left(x_{k}\right)-2 f\left(y_{k}\right)} \frac{f\left(y_{k}\right)}{f^{\prime}\left(x_{k}\right)}, \\
x_{k+1} & =z_{k}-\frac{f\left(z_{k}\right)}{2 f\left[y_{k}, x_{k}\right]-f^{\prime}\left(x_{k}\right)+f\left[z_{k}, x_{k}, x_{k}\right]\left(z_{k}-y_{k}\right)}\left(1+w_{k}+2 v_{k}-2 u_{k}^{3}+\frac{2}{5} \frac{f\left(z_{k}\right)}{f^{\prime}\left(x_{k}\right)}\right),
\end{aligned}
$$

where $u_{k}=\frac{f\left(y_{k}\right)}{f\left(x_{k}\right)}, v_{k}=\frac{f\left(z_{k}\right)}{f\left(x_{k}\right)}$ and $w_{k}=\frac{f\left(z_{k}\right)}{f\left(y_{k}\right)}$.

In the numerical test made, variable precision arithmetics has been used, with 4000 digits of mantissa in Matlab R2011b. Some reference orbits have been used in the test, that can be found in [14]. As orbital elements of each one of the test orbits are known, the vector position in the instants $t_{1}$ and $t_{2}$ have been re-calculated with 3998 exact digits. Then, our aim is gain from these positions, the orbital elements, with a precision as high as possible:

- Test Orbit I has the position vectors

$$
\overrightarrow{r_{1}} \approx[2.46080928705339,2.04052290636432,0.14381905768815]
$$

and

$$
\overrightarrow{r_{2}} \approx[1.98804155574820,2.50333354505224,0.31455350605251],
$$

measured in Earth radius (e.r.) at the instants $t_{1}=0$ and $t_{2}=0.01044412000000$ julian days (J.D.). The orbital elements corresponding to the geometry of the orbit are the semimajor axis $a=4$ e.r., the eccentricity $e=0.2$, the epoch of the perigee $T_{0}=0 h 0 \mathrm{~m} 0 \mathrm{~s}$, and the Euler angles which fit the orbit in space are the Right Ascension of the ascending node, $\Omega=30^{\circ}$, the argument of the perigee $\omega=10^{\circ}$ and the inclination of the orbit $i=15^{\circ}$.

- Test Orbit II. Position vectors and times:

$$
\begin{gathered}
\overrightarrow{r_{1}} \approx[-1.75981065999937,1.68112802634201,1.16913429510899] \text { e.r., } t_{1}=0 \text { J.D., } \\
\overrightarrow{r_{2}} \approx[-2.23077219993536,0.77453561301361,1.34602197883025] \quad \text { e.r., } t_{2}=0.01527809 \text { J.D., }
\end{gathered}
$$

Orbital elements: $\Omega=80^{\circ}, \omega=60^{\circ}, i=30^{\circ}, a=3$ e.r., $e=0.1, T_{0}=0 h 0 \mathrm{~m} 0 \mathrm{~s}$.

- Test Orbit III. Position vectors and times:

$$
\begin{gathered}
\overrightarrow{r_{1}} \approx[0.41136206679761,-1.66250000000000,0.82272413359522] \text { e.r., } t_{1}=0 \text { J.D., } \\
\overrightarrow{r_{2}} \approx[0.97756752977209,-1.64428006097667,-0.04236299091612] \quad \text { e.r., } t_{2}=0.01316924 \text { J.D., }
\end{gathered}
$$

Orbital elements: $\Omega=120^{\circ}, \omega=150^{\circ}, i=60^{\circ}, a=2$ e.r., $e=0.05, T_{0}=0 h 0 \mathrm{~m} 0 \mathrm{~s}$. 
By using these test positions vectors and times, we will consider that the solution provided by Newton's scheme with a required accuracy of $10^{-3998}$ is the exact solution $y_{e x}$. Then we will compare the exact error at the first three iterations of the proposed eighth-order method M8 and the known schemes K8, F8, D8 and S8. We also include, in Tables 1 to 3, the computational order of convergence (COC) (see [22]), in order to check the computational efficiency of the schemes related to their theoretical rate of convergence. This index is evaluated by the formula

$$
C O C \approx \frac{\log \left|\left(x_{k+1}-\xi\right) /\left(x_{k}-\xi\right)\right|}{\log \left|\left(x_{k}-\xi\right) /\left(x_{k-1}-\xi\right)\right|} .
$$

\begin{tabular}{|c|c|c|c|c|c|c|}
\hline Scheme & Fixed Point & K8 & F8 & D8 & S8 & $M_{1} 8$ \\
\hline$\left|y_{1}-y_{e x}\right|$ & 0.006399 & $8.572 \mathrm{e}-25$ & $1.717 \mathrm{e}-18$ & $1.459 \mathrm{e}-24$ & $1.2369276 \mathrm{e}-24$ & $1.244 \mathrm{e}-24$ \\
\hline$\left|y_{2}-y_{e x}\right|$ & $8.157 \mathrm{e}-5$ & $8.642 \mathrm{e}-200$ & $2.389 \mathrm{e}-96$ & $1.105 \mathrm{e}-197$ & $2.503241 \mathrm{e}-198$ & $2.636 \mathrm{e}-198$ \\
\hline$\left|y_{3}-y_{e x}\right|$ & $1.03 \mathrm{e}-6$ & $9.217 \mathrm{e}-1600$ & $1.246 \mathrm{e}-485$ & $1.198 \mathrm{e}-1582$ & $7.043196 \mathrm{e}-1588$ & $1.075 \mathrm{e}-1587$ \\
\hline COC & 1.0 & 8.0 & 5.0 & 8.0 & 8.0 & 8.0 \\
\hline
\end{tabular}

Table 1: Comparison of modified-Gauss schemes for Reference Orbit I

The different test orbits have been chosen with an important property: the increasing angle $\nu_{2}-\nu_{1}$. It measures the spread in the observations and, by the design of Gauss' procedure, it induces instability in the system when it gets higher. The difference between the true anomalies of the observations is, for the test orbits I to III, $\nu_{2}-\nu_{1}=12.23^{\circ}, \nu_{2}-\nu_{1}=22.06^{\circ}$ and $\nu_{2}-\nu_{1}=31.46^{\circ}$, respectively. It can be observed in Tables 1 to 3 that, when the spread of the observations increases, the precision obtained in the calculations per step reduces in the same rate for any eighth-order method.

\begin{tabular}{|c|c|c|c|c|c|c|}
\hline Scheme & Fixed Point & K8 & F8 & D8 & S8 & $M_{1} 8$ \\
\hline$\left|y_{1}-y_{e x}\right|$ & 0.02314 & $4.650 \mathrm{e}-18$ & $1.327 \mathrm{e}-13$ & $4.57 \mathrm{e}-18$ & $3.8834479 \mathrm{e}-18$ & $2.425 \mathrm{e}-18$ \\
\hline$\left|y_{2}-y_{e x}\right|$ & 0.001055 & $1.053 \mathrm{e}-143$ & $8.253 \mathrm{e}-70$ & $1.216 \mathrm{e}-143$ & $2.8055145 \mathrm{e}-144$ & $4.214 \mathrm{e}-146$ \\
\hline$\left|y_{3}-y_{e x}\right|$ & $4.652 \mathrm{e}-5$ & $7.296-1149$ & $7.694 \mathrm{e}-351$ & $3.061 \mathrm{e}-1148$ & $2.0814831 \mathrm{e}-1153$ & $3.508 \mathrm{e}-1168$ \\
\hline COC & 1.0 & 8.0 & 5.0 & 8.0 & 8.0 & 8.0 \\
\hline
\end{tabular}

Table 2: Comparison of modified-Gauss schemes for Reference Orbit II

It is clear that the application of high-order schemes to the problem of preliminary orbit calculation by Gauss procedure gets an important success, as the gain in speed and the precision obtained in the calculations are increased. Nevertheless, the behavior of all the high-order schemes are not the same. The great complexity of the calculations involved in the problem and the own stability of each iterative scheme are important factors to take into account: let us note that Khan's procedure is not appropriated for this particular problem, as it converges more slowly than the rest of scheme, being a good method with theoretical order of convergence eight.

\begin{tabular}{|c|c|c|c|c|c|c|}
\hline Scheme & Fixed Point & K8 & F8 & D8 & S8 & $M_{1} 8$ \\
\hline$\left|y_{1}-y_{e x}\right|$ & 0.05052 & $6.249 \mathrm{e}-14$ & $9.195 \mathrm{e}-11$ & $2.6 \mathrm{e}-14$ & $2.1653574 \mathrm{e}-14$ & $4.962 \mathrm{e}-15$ \\
\hline$\left|y_{2}-y_{e x}\right|$ & 0.004936 & $2.263 \mathrm{e}-109$ & $1.888 \mathrm{e}-54$ & $1.753 \mathrm{e}-112$ & $3.3635496 \mathrm{e}-113$ & $7.299 \mathrm{e}-119$ \\
\hline$\left|y_{3}-y_{e x}\right|$ & 0.0004492 & $6.695 \mathrm{e}-873$ & $6.897 \mathrm{e}-273$ & $7.503 \mathrm{e}-898$ & $1.1401025 \mathrm{e}-903$ & $1.6 \mathrm{e}-949$ \\
\hline COC & 1.0 & 8.0 & 5.0 & 8.0 & 8.0 & 8.0 \\
\hline
\end{tabular}

Table 3: Comparison of modified-Gauss schemes for Reference Orbit III

Let us note that the precision of the orbital elements calculated with the third estimation provided by any eighth-order method is total, as all the 4000 decimal digits of the solution considered as exact are reached with only three iterations. 


\section{Numerical results}

In this section we show the convergence behavior of the proposed methods $M_{1} 8$ and $M_{2} 8$. In the numerical test made, variable precision arithmetics has been used, with 4000 digits of mantissa in Matlab R2010a. In our numerical experiments we compare our schemes with Newton's method (NM), Steffensen's method (SM), Ostrowski's method (OM), and several iterative procedures, quoted below.

KWM [3]

$$
\begin{aligned}
y_{k} & =x_{k}-\frac{f\left(x_{k}\right)}{f^{\prime}\left(x_{k}\right)}, \\
z_{k} & =y_{k}-H_{1} \frac{f\left(y_{k}\right)}{f^{\prime}\left(x_{k}\right)}, \\
x_{k+1} & =z_{k}-\left[\left(1+H_{1}\right)^{2}+\left(1+4 H_{1}\right) H_{2}\right] \frac{f\left(z_{k}\right)}{f^{\prime}\left(x_{k}\right)},
\end{aligned}
$$

where $H_{1}=\frac{f\left(x_{k}\right)}{f\left(x_{k}\right)-2 f\left(y_{k}\right)}$ and $H_{2}=\frac{f\left(z_{k}\right)}{f\left(y_{k}\right)-3 f\left(z_{k}\right)}$.

CTVM [7]

$$
\begin{aligned}
y_{k} & =x_{k}-\frac{f\left(x_{k}\right)}{f^{\prime}\left(x_{k}\right)}, \\
z_{k} & =y_{k}-H \frac{f\left(y_{k}\right)}{f^{\prime}\left(x_{k}\right)}, \\
x_{k+1} & =u_{k}-3 \frac{u_{k}-z_{k}}{y_{k}-x_{k}} \frac{f\left(z_{k}\right)}{f^{\prime}\left(x_{k}\right)},
\end{aligned}
$$

where $H=\frac{f\left(x_{k}\right)}{f\left(x_{k}\right)-2 f\left(y_{k}\right)}, u_{k}=z_{k}-(H+T / 2)^{2} \frac{f\left(z_{k}\right)}{f^{\prime}\left(x_{k}\right)}$ and $T=\frac{f\left(z_{k}\right)}{f\left(y_{k}\right)-2 f\left(z_{k}\right)}$.

ZLHM [8]

$$
\begin{aligned}
y_{k} & =x_{k}-\frac{f\left(x_{k}\right)}{f\left[x_{k}, z_{k}\right]}, \quad z_{k}=x_{k}+\gamma f\left(x_{k}\right), \\
u_{k} & =y_{k}-\frac{f\left(y_{k}\right)}{f\left[y_{k}, x_{k}\right]+f\left[y_{k}, x_{k}, z_{k}\right]\left(y_{k}-x_{k}\right)}, \\
x_{k+1} & =u_{k}-\frac{f\left(u_{k}\right)}{f\left[u_{k}, y_{k}\right]+f\left[u_{k}, y_{k}, x_{k}\right]\left(u_{k}-y_{k}\right)+f\left[u_{k}, y_{k}, x_{k}, z_{k}\right]\left(u_{k}-y_{k}\right)\left(u_{k}-x_{k}\right)},
\end{aligned}
$$

SKM [11]

$$
\begin{aligned}
y_{k} & =x_{k}-\frac{f\left(x_{k}\right)}{f\left[x_{k}, z_{k}\right]}, \quad z_{k}=x_{k}-f\left(x_{k}\right), \\
u_{k} & =y_{k}-H \frac{f\left(x_{k}\right)}{f\left[x_{k}, z_{k}\right]}, \\
x_{k+1} & =u_{k}-G \frac{f\left(u_{k}\right)}{f\left[x_{k}, z_{k}\right]},
\end{aligned}
$$

where $H=1+w_{1}\left(x_{k}\right)+w_{2}\left(x_{k}\right), w_{1}\left(x_{k}\right)=\frac{f\left(y_{k}\right)}{f\left(x_{k}\right)}, w_{2}\left(x_{k}\right)=\frac{f\left(y_{k}\right)}{f\left(z_{k}\right)}, G=g_{1}+g_{2}+g_{3}, g_{1}=1+(2-$ $\left.f\left[x_{k}, z_{k}\right]\right) w_{1}\left(x_{k}\right)+\left(1-f\left[x_{k}, z_{k}\right]\right) w_{1}\left(x_{k}\right)^{2}, g_{2}=\left(4-f\left[x_{k}, z_{k}\right]\left(6+f\left[x_{k}, z_{k}\right]\left(-4+f\left[x_{k}, z_{k}\right]\right)\right)\right) w_{1}\left(x_{k}\right)^{3}, g_{3}=$ $w_{3}\left(x_{k}\right)+w_{3}\left(x_{k}\right)^{2}+\left(4-2 f\left[x_{k}, z_{k}\right]\right) w_{4}\left(x_{k}\right), w_{3}\left(x_{k}\right)=\frac{f\left(u_{k}\right)}{f\left(y_{k}\right)}$ and $w_{4}=\frac{f\left(u_{k}\right)}{f\left(z_{k}\right)}$.

Table 4 shows the expression of the test functions, the roots with sixteen significant digits and the initial approximation $x_{0}$ which is the same for all methods. Displayed in Table 5 is the number of iterations (It), the computational order of convergence (COC), the absolute value of the function $\left|f\left(x_{k}\right)\right|$ at the last iteration and root obtained at each method $\xi$.

These numerical results shown are in accordance with the efficiency analysis the show in Theorem 1 and Theorem 2 presented in Section 2. Both the numerical results, such as the dynamic analysis show that derivativefree methods are more sensitive to the initial approximation comparing them with those that use derivatives. 
Table 4: Test functions and their roots

\begin{tabular}{|c|c|c|}
\hline Test functions & Roots & Starting points \\
\hline$f_{1}=\sqrt{x^{4}+8} \sin \left(\frac{\pi}{x^{2}+2}\right)+\frac{x^{3}}{x^{4}+1}-\sqrt{6}+\frac{8}{17}$ & $\xi_{1}=-2$ & $x_{0}=-1.8$ \\
& $\xi_{2} \approx-1.149212674609088$ & \\
\hline$f_{2}=x \exp \left(x^{2}\right)-\sin \left(x^{2}\right)+3 \cos (x)+5$ & $\xi \approx-1.201576112092293$ & $x_{0}=2$ \\
\hline$f_{3}=\sqrt{x^{2}+2 x+5}-2 \sin (x)-x^{2}+3$ & $\xi_{1} \approx+2.3319676558839640$ & $x_{0}=1$ \\
\hline$f_{4}=x^{4}+\sin \left(\frac{\pi}{x^{2}}\right)-5$ & $\xi_{2} \approx-2.573166514902827$ & \\
\hline$f_{5}=\left(\sin (x)-\frac{x}{2}\right)^{2}$ & $\xi_{1} \approx+1.414213562373095$ & $x_{0}=2$ \\
\hline & $\xi_{2} \approx-1.414213562373095$ & \\
\hline
\end{tabular}

\section{References}

[1] J. F. Traub, Iterative methods for the solution of equations, Chelsea Publishing Company, New York, 1977.

[2] M. Petković, B. Neta, L. Petković and J. Džunić, Multipoint methods for solving nonlinear equations, Academic Press, 2012.

[3] J. Kou and X. Wang, Some improvements of Ostrowski's method, Appl. Math. L., 23 (2010) 92-96.

[4] Y.I. Kim, A triparametric family of theree-step optimal eighth-order multipoint iterative methods for solving nonlinear equations, Inter. J. of Comp. Math., 89 (2012) 1051-1059.

[5] Y. Khan, M. Fardi and K. Sayevand, A new general eighth-order family of iterative methods for solving nonlinear equations, Appl. Math. L., 25 (2012) 2262-2266.

[6] J. R. Sharma and R. Sharma, A new family of modified Ostrowski's methods with accelerated eighth-order convergence, Numer. Algor., 54 (2010) 445-458.

[7] A. Cordero, J.R. Torregrosa and M. Vassileva, Tree-step iterative method with a optimal eighth order of convergence, J. of Comp. and Appl. Math, 235 (2011) 3189-3194.

[8] Q. Zheng, J. Li and F. Huang, An optimal Steffensen-type family for solving nonlinear equations, Appl. Math. and Comput., 217 (2011) 9592-9597.

[9] H. Ren, Q. Wu and W. Bi, A class of two-step Steffensen type methods with fourth-order convergence, Appl. Math. and Comput., 209 (2009) 206-210.

[10] Z. Liu, Q. Zheng and P. Zhao, A variant of Steffensen's method of fourth-order convergence and its applications, Appl. Math. and Comput., 216 (2010) 1978-1983.

[11] F. Soleymani and S.K. Khattri, Finding simple roots by seventh- and eighth-order derivative-free methods, Int. J. Math. Models Meth. Appl. Sci., 1 (2012) 45-52.

[12] M.S. Petković, S. Ilić and J. Džunić, Derivative free two-point methods with and without memory for solving nonlinear equations, Appl. Math. and Comput., 217 (2010) 1887-1895.

[13] S. Artidiello, F. Chicharro, A. Cordero and J. R. Torregrosa, Local convergence and dynamical analysis of a new family of optimal fourth-order iterative methods, Inter. J. of Comp. Math., 90(10) (2013) 2049-2060.

[14] P.R. Escobal. Methods of orbit determination, Robert E. Krieger Publishing Company, 1965.

[15] H. T. Kung and J. F. Traub, Optimal order of one-point and multipoint iteration, J. Assos. Comput. Math., 21 (1974) 643-651.

[16] J. Džunić, M.S. Petković and L.D. Petković, A family of optimal three-point methods for solving nonlinear equations using two parametric functions, Appl. Math. and Comput., 217 (2011) 7612-7619. 


\begin{tabular}{|c|c|c|c|c|c|}
\hline$\stackrel{\infty}{\infty}$ & 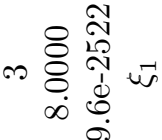 & 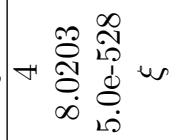 & 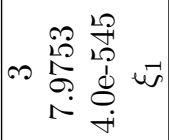 & ن. & 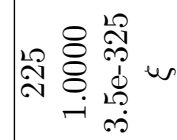 \\
\hline$\sum_{\substack{x \\
\text { in }}}^{1}$ & 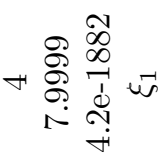 & $\dot{ن}$ & 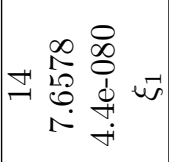 & 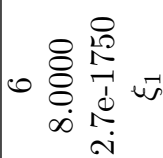 & 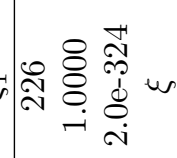 \\
\hline$\sum_{\mathbf{N}}$ & 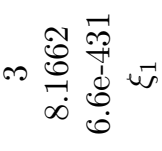 & 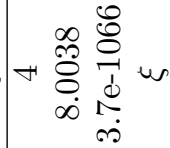 & 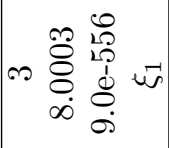 & 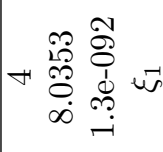 & 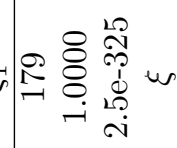 \\
\hline$\underset{\Sigma}{\infty}$ & 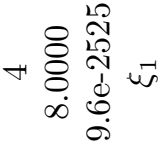 & 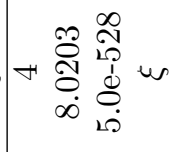 & 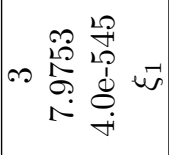 & $\dot{ن}$ & 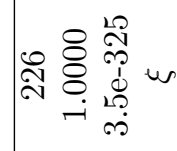 \\
\hline$\sum_{i}^{2}$ & 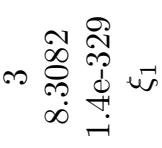 & 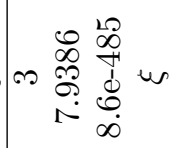 & 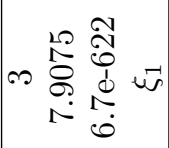 & 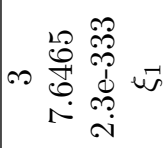 & 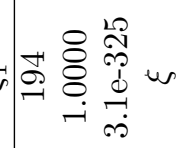 \\
\hline$\sum_{\substack{1 \\
\mid}}^{1}$ & 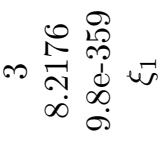 & 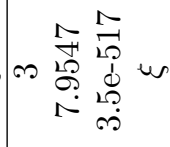 & 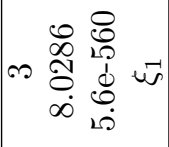 & 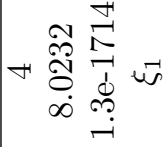 & ن \\
\hline$\sum_{0}^{1}$ & 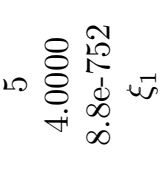 & 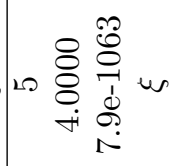 & 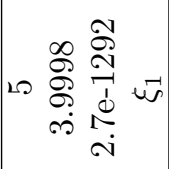 & L & 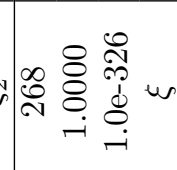 \\
\hline$\sum_{\infty}$ & 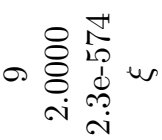 & $\dot{\dot{g}}$ & 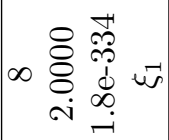 & 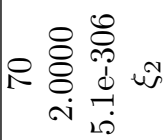 & 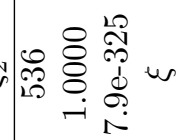 \\
\hline$\sum_{\text {Z }}$ & 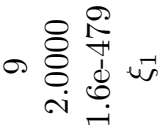 & 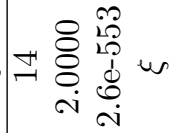 & 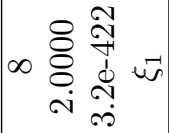 & 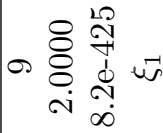 & 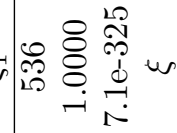 \\
\hline & \pm 它 & 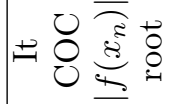 & 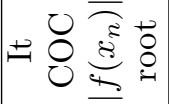 & 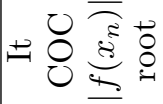 & 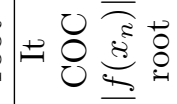 \\
\hline & $\leftrightarrow$ & $\stackrel{N}{N}$ & $\stackrel{\infty}{\stackrel{2}{2}}$ & 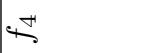 & +20 \\
\hline
\end{tabular}


[17] P. Blanchard, The Dynamics of Newton's Method, Proc. of Symposia in Applied Math., 49 (1994) 139-154.

[18] F. Chicharro, A. Cordero, J.R. Torregrosa, Drawing dynamical and parameters planes of iterative families and methods, The Scientific World Journal Volume 2013 Article ID:780153 11 pages.

[19] J. Džunić, M. Petković, A family of Three-Point methods of Ostrowski's Type for Solving Nonlinear Equations, Jounal of Applied Mathematics ID 425867 (2012), 9 pages, doi:10.1155/2012/425867.

[20] A.M. Ostrowski, Solution of Equations and System of Equations, Prentice-Hall, Englewood Cliffs, NJ, USA, 1964.

[21] F. Soleymani, M. Sharifi, B.S. Mousavi, An Improvement of Ostrowski's and King's Techniques with Optimal Convergence Order Eight, J. Optim. Theory Appl., 153 (2012) 225-236.

[22] S. Weerakoon and T.G.I. Fernando, A variant of Newton's method with accelerated third-order convergence, Appl. Math. L., 13 (2000) 87-93. 